\title{
Computational methods for gas dynamics and compressible multiphase flows
}

\author{
D. Zeidan ${ }^{1}$ (D) H. D. $\mathbf{N g}^{2}(\mathbb{D}$
}

Published online: 27 October 2018

c) Springer-Verlag GmbH Germany, part of Springer Nature 2018

Computational fluid dynamics (CFD) has emerged in the past few decades as a powerful tool in many fields of basic and applied sciences. Together with ever-growing development of technologies in computer hardware, there has been a rapid evolution of CFD techniques and models in recent years. CFD simulation helps researchers to become more efficient, and provides the capability to obtain accurate supplemental information, which is challenging to obtain from experimental measurements. The primary objective of this thematic issue has been to provide representative coverage on recent advances and application of CFD in the field of gas dynamics and compressible multiphase fluid flows. Given the broad nature of CFD research, it is hardly possible to achieve an exhaustive representation of the selected topics in a single journal issue. Nonetheless, this thematic issue brings together a broad spectrum of researchers from different quarters and represents a respected panel that addresses various aspects of CFD modeling and application, from the formulation of new higher-order numerical methods for gas dynamics to the simulations of multiphysics problems involving compressible fluid flows.

Manuscripts in this thematic issue were solicited through a general call and by invitation. It also contains selected papers that were presented at the 11th International Symposium on Numerical Analysis of Fluid Flows, Heat and Mass Transfer-Numerical Fluids 2016 held in Rhodes, Greece, September 19-25, 2016. Submissions were subjected to a rigorous peer-reviewed process following the established standards and policies of the Shock Waves Journal. Each paper was reviewed by at least two internationally recog-

$\triangle$ D. Zeidan

dia.zeidan@gju.edu.jo

H. D. Ng

hoing@encs.concordia.ca

1 School of Basic Sciences and Humanities, German Jordanian University, Amman, Jordan

2 Department of Mechanical, Industrial and Aerospace Engineering, Concordia University, Montreal, QC, Canada nized experts in the field. Twenty-three manuscripts were received in response to the thematic issue announcement, of which eight papers were rejected after peer review and two were withdrawn by the authors following a "major revision" decision. The remaining 13 papers were eventually accepted upon up to three revisions, after a consensus was reached by the referees and the editors. Thus, the rejection rate for the issue is $43 \%$, which is one of the indicators of the high standards applied when preparing the issue.

This thematic issue includes 13 papers which can be grouped into three categories. The first group includes five contributions on the discussion and development of contemporary numerical techniques. Zhu and Shu [1] introduced a new class of fifth-order weighted essentially non-oscillatory (WENO) finite-volume schemes on unstructured meshes and demonstrated its convergence in steady-state calculations, which is often difficult to achieve with high-order WENO schemes. The paper by Margolin [2] gives an interesting fundamental interpretation of the notion of artificial viscosity from the conservation equations of finite scale. This contribution also provides relevant insights into finite-volume method formulations. Soni et al. [3] introduced a promising adaptive multiresolution methodology with a time-varying tolerance strategy by combining a wavelet-based adaptive multiresolution scheme in conjunction with an immersed boundary method for capturing fine-scale features of a class of shock-bluff-body interactions. Compressible flows typically contain shocks and interfaces. To capture these features correctly and overcome difficulties encountered by classical finite-volume approaches, such as spurious oscillations and nonphysical front blurring, a new front tracking method for one-dimensional flow is introduced by Cao et al. [4] based on the Lagrangian method and a simplified Kamm's exact Riemann solver. The work by Re and Guardone [5] assesses for the first time the application of mesh adaptation techniques within the arbitrary Lagrangian-Eulerian framework in the non-ideal compressible fluid dynamic (NICFD) regime.

The second set of papers is concerned with the application of contemporary numerical methods to supersonic 
flows and shock wave-related phenomena. Using an entropygeneration-based artificial viscosity and a high-order explicit discontinuous spectral element method (DSEM), Chaudhuri and Jacobs [6] examined in greater depth the shock wave diffraction over a sharp splitter geometry and revealed new features via a detailed transient flow analysis in terms of dissipation and dilatation. In another important area of shock wave research, Pérez Arroyo et al. [7] analyzed results obtained using large eddy simulation (LES) focusing on the near-field of a non-screeching supersonic under-expanded jet. This detailed work investigates the mechanism of generation of broadband shock-associated noise (BBSAN) by combining different techniques of signal processing such as wavenumber-frequency Fourier analysis, wavelet transform, and conditional averaging. The work by Soni et al. [8] analyzes a Mach 3 supersonic cavity flow simulated using large eddy simulation (LES). Contemporary data-processing techniques of proper orthogonal decomposition (POD) and dynamic mode decomposition (DMD) are used to study the spatial and temporal evolution of a supersonic cavity flow field.

The last part of this thematic issue is devoted to the computation of multiphase flows. Three papers deal with the numerical modeling of condensed phase detonation. Using their recently developed multiphase models, Michael and Nikiforakis [9] examined in their first paper the shockinduced cavity collapse in inert liquid nitromethane, particularly focusing on the evolution of the temperature field in the vicinity of the cavity. The second paper by Michael and Nikiforakis [10] considers the shock-induced cavity collapse in reacting liquid nitromethane, focusing on the chemical reaction activities and flow evolution in hot spots. The contribution by Schwendeman et al. [11] investigates the numerical results using a modified multiscale model to advance the current understanding of detonation initiation in heterogeneous condensed phase explosives by transient piston-induced compaction. This issue is concluded with two papers on multiphase flows, one by Goncalves et al. [12] presenting the development of a four-equation model for the classical shock-induced bubble collapse and one by Kim et al. [13] exploiting the robustness and accuracy of selected numerical methods for simulating multiphase real fluid flows at all speeds, and their suitability to a broad range of engineering applications.

We believe that this thematic issue highlights the recent growing importance of CFD and will make a significant contribution to the community by identifying and discussing several interesting problems in the numerical analysis of gas dynamics and compressible multiphase flows. We wish to thank the authors who submitted their works to this issue and revised their manuscripts to be in line with the high-quality standards of the journal. We would also like to acknowledge the efforts by all referees on reviewing these papers and making it possible to publish this special issue.

\section{References}

1. Zhu, J., Shu, C.W.: Numerical study on the convergence to steady state solutions of a new class of finite volume WENO schemes: triangular meshes. Shock Waves 29(1) (2019). https://doi.org/10.1 007/s00193-018-0833-1

2. Margolin, L.G.: The reality of artificial viscosity. Shock Waves 29(1) (2019). https://doi.org/10.1007/s00193-018-0810-8

3. Soni, V., Hadjadj, A., Roussel, O.: On the use of adaptive multiresolution method with time-varying tolerance for compressible fluid flows. Shock Waves 29(1) (2019). https://doi.org/10.1007/s00193017-0777-x

4. Cao, Y., Wang, Z., Hong, T.: A front tracking method capturing field features accurately for one-dimensional flows. Shock Waves 29(1) (2019). https://doi.org/10.1007/s00193-018-0845-x

5. Re, B., Guardone, A.: An adaptive ALE scheme for non-ideal compressible fluid dynamics over dynamic unstructured meshes. Shock Waves 29(1) (2019). https://doi.org/10.1007/s00193-018-0840-2

6. Chaudhuri, A., Jacobs, G.B.: Dynamics of shock wave diffraction over sharp splitter geometry using entropy-based artificial viscosity method. Shock Waves 29(1) (2019). https://doi.org/10.1007/s001 93-017-0767-z

7. Pérez Arroyo, C., Daviller, G., Puigt, G., Airiau, C., Moreau, S.: Identification of temporal and spatial signatures of broadband shock-associated noise. Shock Waves 29(1) (2019). https://doi.or g/10.1007/s00193-018-0806-4

8. Soni, R.K., Arya, N., De, A.: Modal decomposition of turbulent supersonic cavity. Shock Waves 29(1) (2019). https://doi.org/10.1 007/s00193-018-0836-y

9. Michael, L., Nikiforakis, N.: The evolution of the temperature field during cavity collapse in liquid nitromethane. Part I: inert case. Shock Waves 29(1) (2019). https://doi.org/10.1007/s00193-018-0 $802-8$

10. Michael, L., Nikiforakis, N.: The evolution of the temperature field during cavity collapse in liquid nitromethane. Part II: reactive case. Shock Waves 29(1) (2019). https://doi.org/10.1007/s00193-018-0 803-7

11. Gambino, J.R., Schwendeman, D.W., Kapila, A.K.: Numerical study of multiscale compaction-initiated detonation. Shock Waves 29(1) (2019). https://doi.org/10.1007/s00193-018-0805-5

12. Goncalves, E., Hoarau, Y., Zeidan, D.: Simulation of shockinduced bubble collapse using a four-equation model. Shock Waves 29(1) (2019). https://doi.org/10.1007/s00193-018-0809-1

13. Kim, H., Choe, Y., Kim, H., Min, D., Kim, C.: Methods for compressible multiphase flows and their applications. Shock Waves 29(1) (2019). https://doi.org/10.1007/s00193-018-0829-x

Publisher's Note Springer Nature remains neutral with regard to jurisdictional claims in published maps and institutional affiliations. 